\title{
Harris-type van der Waals density functional scheme
}

\author{
Kristian Berland, Elisa Londero, Elsebeth Schröder," and Per Hyldgaard \\ Microtechnology and Nanoscience, MC2, Chalmers University of Technology, SE-412 96 Göteborg, Sweden
}

(Received 18 March 2013; revised manuscript received 26 May 2013; published 18 July 2013)

\begin{abstract}
Biomolecular systems that involve thousands of atoms are difficult to address with standard density functional theory (DFT) calculations. With the development of sparse-matter methods such as the van der Waals density functional (vdW-DF) method [M. Dion et al., Phys. Rev. Lett. 92, 246401 (2004)], it is now possible to include the dispersive forces in DFT which are necessary to describe the cohesion and behavior of these systems. vdW-DF implementations can be as efficient as those for traditional DFT. Yet, the computational costs of self-consistently determining the electron wave functions and hence the kinetic-energy repulsion still limit the scope of sparse-matter DFT. We propose to speed up sparse-matter calculations by using the Harris scheme [J. Harris, Phys. Rev. B 31, 1770 (1985)]; that is, we propose to perform electronic relaxations only for separated fragments (molecules) and use a superposition of fragment densities as a starting point to obtain the total energy non-self-consistently. We evaluate the feasibility of this approach for an adaption of the Harris scheme for non-self-consistent vdW-DF (sfd-vdW-DF). We study four molecular dimers with varying degrees of polarity and find that the sfd scheme accurately reproduces standard non-self-consistent vdW-DF for van der Waals dominated systems but is less accurate for those dominated by polar interactions. Results for the S22 set of typical organic molecular dimers are promising.
\end{abstract}

DOI: 10.1103/PhysRevB.88.045431

PACS number(s): 31.15.E-, 31.15.ae, 71.15.Nc

\section{INTRODUCTION}

Density functional theory (DFT) is one of the best and most reliable condensed-matter tools for studying molecular, surface, bulk, and compound properties of matter. ${ }^{1}$ Standard implementations, using the local density approximation (LDA) or the generalized gradient approximation (GGA) for the exchange-correlation energy, accurately describe the binding in regions characterized by high-electron density. Soft- and sparse-matter systems have internal voids or low-electrondensity regions where the van der Waals (vdW) forces, also called London dispersion forces, ${ }^{2}$ are essential. While neither LDA nor GGA capture the truly nonlocal correlation effects that underpin these forces, ${ }^{3-7}$ in the last decade both vdW-extended DFT (Refs. 5 and 8-11) and regular nonlocal exchange-correlation functionals including $\mathrm{vdW}$ forces have been developed. ${ }^{12-19}$ Methods of the first group are often atom centered and generally require use of damping functions, while those of the second group fit within the regular DFT formulation.

The vdW density functional (vdW-DF) method ${ }^{6,7,13,14,19-21}$ provides a framework for approximating the exchangecorrelation energy $E_{\mathrm{xc}}[n]$. It starts from the adiabaticconnection formula ${ }^{22,23}$ (ACF) and a plasmon-pole approximation ${ }^{6,13}$ that captures a collectivity effect that reflects a broader density variation. ${ }^{13,20,21}$ Within this framework, several general-purpose nonempirical functionals ${ }^{12,13,19}$ have been developed. In the recent explicit functional versions, called vdW-DF1 (Refs. 13 and 14) and vdW-DF2 (Ref. 19), the nonlocal energy $E_{c}^{\mathrm{nl}}[n]$ is expressed as a double integral over the density, weighted by a kernel. The vdW-DF1 and vdW-DF2 are total energy functionals that include nonlocal correlation in addition to kinetic and electrostatic terms as well as LDA correlation and a specified exchange functional. ${ }^{24,25}$ Their nonempirical designs suggest that the vdW-DFs can achieve a good transferability across system type, length scales, charging states, and binding morphologies.
The performance of vdW-DF has been tested for bulk $\mathrm{k}^{26-31}$ and layered ${ }^{7,12,32,33}$ systems, for molecular and atomic absorption, ${ }^{34-36}$ for adsorption, ${ }^{37-50}$ and self-assembly on surfaces ${ }^{51-53}$ and for molecular systems ${ }^{54-58}$ Self-consistent (sc) vdW-DF calculations ${ }^{14}$ can be used to calculate stress within periodic unit cells ${ }^{59}$ and guide atomic relaxation. Alternative exchange functionals have been developed with the aim of improving the vdW-DF's performance. ${ }^{60-62}$

Effective algorithms ${ }^{63-67}$ have allowed self-consistent (sc) vdW-DF implementations to have a computational efficiency similar to that of GGA calculations. ${ }^{45}$ For very large systems, typical of biomolecular-interaction problems, real-space vdWDF implementations ${ }^{64-67}$ permit an order- $N$ scaling of the evaluation of $E_{c}^{\mathrm{nl}}[n]$, as discussed in Ref. 29. Nevertheless, the scope of sparse-matter DFT calculations is limited if huge numbers of atoms are involved. Once we reach thousands of atoms, both first-principles and vdW-extended DFT calculations are limited by memory requirements and computational costs of the wave-function evaluation. The challenge is thus to compute the steric hindrance effects that are described by the kinetic-energy repulsion.

In this paper, we propose to reduce the computational cost of evaluating the kinetic energy of sparse-matter systems by building on the Harris scheme. ${ }^{6-73}$ This approach is tested for an adaption of the Harris scheme for non-self-consistent (nsc) vdW-DF calculations. Specifically, we use a superposition of fragment (electron) densities (sfd) as a starting point for non-self-consistently evaluating the total energy of vdW-DF (sfd-vdW-DF). In the sfd framework, the charge densities of the fragments can be obtained with some other method than the method used to calculate the exchange-correlation energy (here vdW-DF). In the regular Harris scheme, the same functional is used to generate the fragment densities and calculate the exchange-correlation energy.

Sfd schemes can also be developed for other sparse-matter methods as for DFT-D. ${ }^{10}$ We only test vdW-DF here for 
two reasons. First, unlike DFT-D, the nonlocal correlation of vdW-DF is strongly sensitive to density variations in the low-density regions. ${ }^{74}$ Thus, the vdW interaction is more prone to be affected by the use of sfd-vdW-DF in place of regular sc-vdW-DF. Further, if the sfd-vdW-DF scheme reproduces sc-vdW-DF results, it indicates that other sparse-matter sfd schemes may also be used in place of the regular DFT method. Second, our work is motivated by a desire to develop methods suitable for describing huge biomolecular systems, such as the DNA interaction problem. ${ }^{75}$ For such a problem, we need to describe the molecular recognition (the noncovalent matching of base pairs and other biomolecules, and packing of our genome) in a solution that contains counter ions that give rise to charging states. Being a parameter-free nonlocal density functional, we believe vdW-DF has an inherent advantage for capturing the change in $\mathrm{vdW}$ response that arises due to such charging effects. The scheme may be particularly useful for systems consisting of many organic molecules bound dominantly by vdW forces, such as C60 clusters.

In this paper, we formally analyze and test the sfd-vdW-DF scheme for molecular systems. The performance is evaluated for the S22 benchmark set ${ }^{76}$ and for four selected systems with a varying degree of static polarizations and size. For the four systems, we also test how well the sfd scheme reproduces the part of the interaction that arises at the semilocal GGA level. Our formal analysis shows that while a regular Harris scheme reproduces the energy of full sc calculations to second order in binding-induced density shift, $\delta n=n_{\mathrm{sc}}-n_{\mathrm{sfd}}$, the sfd scheme only reproduces the energy to linear order. We also find that the linear term $\delta n$ is weighted by the change in the effective Kohn-Sham (KS) potential that occurs as we go from one functional (here a GGA) to another (here a vdW-DF). The sfd-vdW-DF scheme may thus be broadly applicable in the absence of large static dipoles.

The paper is organized as follows. In Sec. II, we present the Harris and sfd schemes, discuss sfd-vdW-DF schemes, and illustrate the feasibility of the approach. We also briefly discuss the formal error that arises in sfd schemes, which is derived in the Appendix. The computational details are presented in Sec. III. Section IV presents an analysis of four model systems, selected to build insight into the performance of sfd-vdWDF. Section V presents details and discusses the results for the S22-based benchmarking of the sfd-vdW-DF method. In Sec. VI, we discuss the computational efficiency of the method. The final section contains a summary and outlook.

\section{HARRIS-TYPE EVALUATION SCHEMES}

\section{A. Recipe}

The (regular) Harris scheme and the more general sfd scheme take the following steps to calculate the total energy for a sparse-matter system:

1. Self-consistently calculate the density $n_{i=1,2, \ldots}$ of each individual building block $i$. In sparse-matter DFT, a building block may correspond to a molecule, a component of a supramolecular system.

2. Construct a density $n_{\text {sfd }}=\sum_{i} n_{i}$ as a superposition of the densities of the building blocks. This is illustrated in the upper panel of Fig. 1.
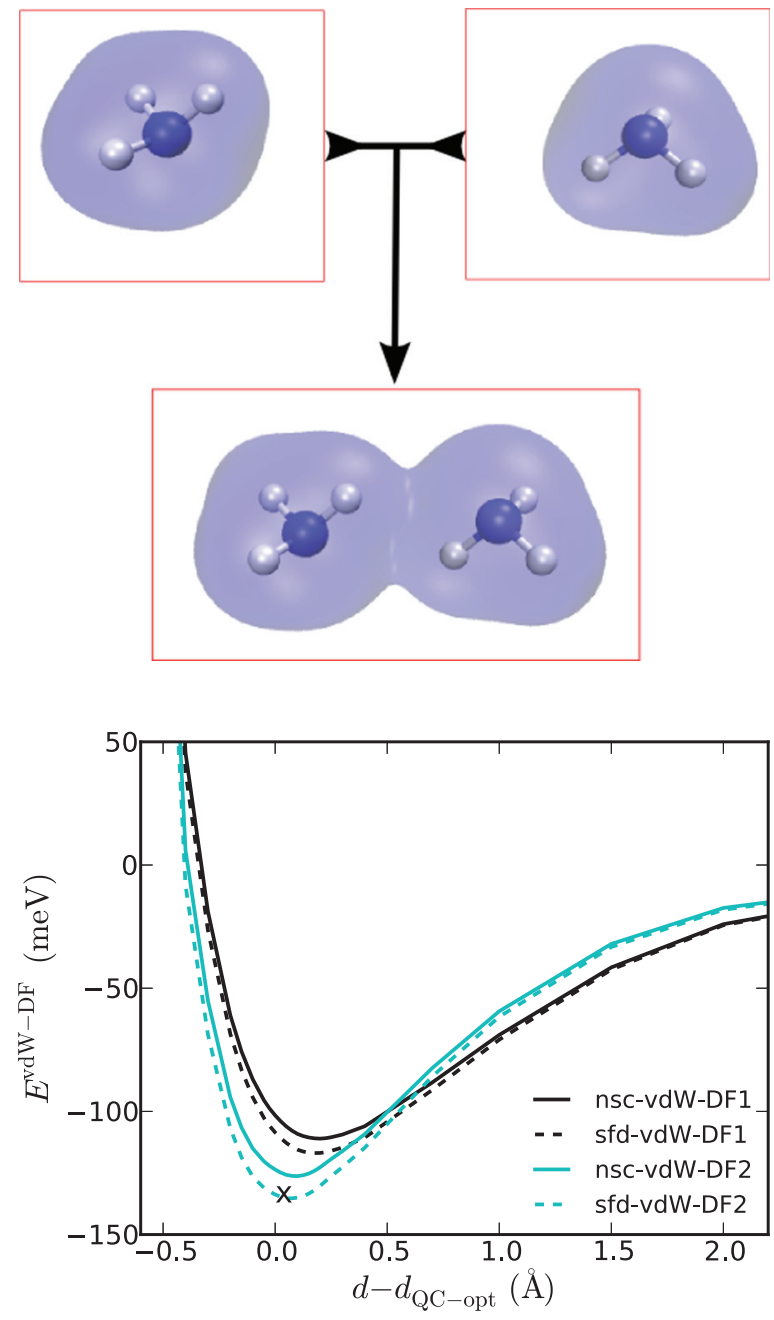

FIG. 1. (Color online) Illustration and test of the sfd scheme using vdW-DF. The upper panel shows a schematic of the superposition of two electron densities for the ammonia $\left(\mathrm{NH}_{3}\right)$ dimer, a member of the S22 benchmark set (Ref. 76). The lower panel shows corresponding binding-energy curves. The dark (light) solid curve indicates the results for non-self-consistent vdW-DF1 (Ref. 13) [vdW-DF2 (Refs. 19 and 29)]. The dashed dark (light) curve indicates the results of sfd-vdW-DF1 (sfd-vdW-DF2). The cross identifies the binding energy and separation as obtained for the original, fully self-consistent vdW-DF2 study (Ref. 19).

3. Use the new density $n_{\text {sfd }}$ as input to calculate the total energy of the system at the Kohn-Sham level without updating the density. The result $E_{\text {sfd }}$ serves as an approximation for the self-consistently obtained total energy $E_{\text {sc }}$.

The last step, which in our case is taken care of within the DACAPO code (Ref. 77) and an external evaluation of the nonlocal correlation energy, ${ }^{26,29,56,67}$ entails constructing the effective single-particle potential $V_{\text {eff,sfd }}(\mathbf{r})=V_{\text {eff }}\left[n_{\text {sfd }}\right](\mathbf{r})$ that corresponds to $n_{\text {sfd. }}$. Further, without updating the density, we calculate the eigenvalues corresponding to the potential $V_{\text {eff,sfd }}(\mathbf{r})$. These eigenvalues are in turn used to provide a sfd estimate of the total energy $E_{\text {sfd. }}{ }^{68}$

If the same functional is used to estimate the total energy as was used to generate the density of the individual building blocks $n_{i}$, we are using a regular Harris scheme ${ }^{68,70,78}$ and 
obtain $E_{\text {sfd }} \rightarrow E_{\text {Harris }}$. If the total exchange-correlation energy is calculated with a different functional than the one used to generate the densities and kinetic energy we are using the more general sfd scheme. If we are using the sfd-vdW-DF scheme with only one component per unit cell $n_{\text {sfd }}=n_{1}$, we are doing nsc-vdW-DF calculations, evaluating $E_{\mathrm{nsc}}^{\mathrm{vdW}-\mathrm{DF}}$.

\section{B. sfd-vdW-DF scheme}

A regular Harris scheme using a vdW-DF is applicable if the fully sc density solution $n_{\text {sc }}$ does not differ significantly from the input superposition density $n_{\text {sfd. }}$. The original study by Harris, ${ }^{68}$ working with LDA, shows that the scheme works reasonably well even for describing covalent bonds between atoms in some molecules. It should be even better suited to describe supramolecular systems, where we can expect charge relocations to be small. The Harris scheme is today often used in band-structure calculations in DFT to provide accurate wave functions and eigenvalues for a given $V_{\text {eff,sfd }}(\mathbf{r})$. We are here interested in the Harris scheme for its original purpose, to provide an efficient but approximate account of interactions in DFT.

We assess the feasibility of the more general sfd scheme for vdW-DF, relying on input densities calculated at the GGA level. The regular Harris scheme for vdW-DF is not tested because we currently lack access to a code with both a self-consistent implementation of vdW-DF and where an externally defined density can be used as input. A benefit of the more general sfd scheme is that it lends itself more naturally to testing different kinds of codes and calculations at various levels of sophistication. We also note that if sfd-vdW-DF calculations accurately reproduce sc results for a class of systems, the same is likely the case for a regular Harris-based vdW-DF scheme.

Figure 1 gives an example of a test of the feasibility of sfdvdW-DF calculations. It compares the binding in an ammonia dimer and shows that the sfd-vdW-DF2 result agrees well with the binding predicted by sc-vdW-DF2 (indicated by a cross).

Figure 2 summarizes our assessment of the sfd-vdW-DF scheme for the S22 benchmark set of organic molecular dimers, as further detailed in Sec. V. It testifies a significant robustness across the $\mathrm{S} 22$ benchmark set. ${ }^{76}$ It shows that the sfd-vdW-DF scheme can be as reliable as the often-used nsc-vdW-DF evaluation. ${ }^{13,26,29,56}$ Thus, the sfd framework represents a promising approach to speed up vdW-DF studies and, by extension, other sparse-matter studies of large biomolecular interaction problems at a limited cost of accuracy.

\section{Formal error estimate of the sfd scheme}

The error introduced by using the (regular) Harris scheme is second order in the density shift $\delta n=n_{\mathrm{sc}}-n_{\mathrm{sfd}}$. In the more general sfd scheme, a linear error term arises as the functional " $g$ " used to generate the input density $n_{i}$, the effective potential, and the single-particle KS energies do not match to the functional " $v$ " used to calculate the exchange-correlation energy. For sfd calculations in this paper, the " $g$ " functional is a GGA and " $v$ " is a vdW-DF.
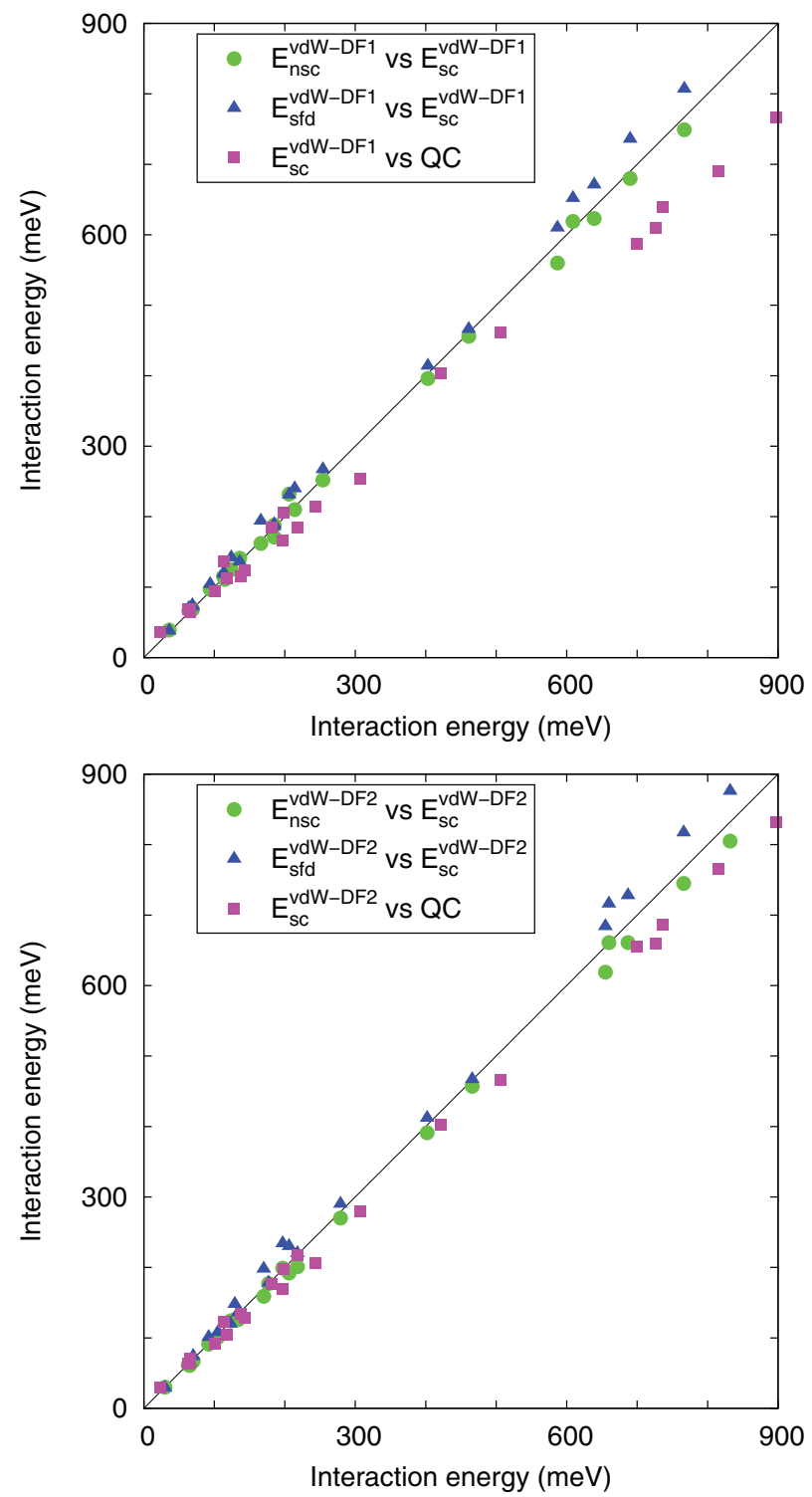

FIG. 2. (Color online) Organic-molecule assessment of the Harris-type sfd scheme using vdW-DF. Results for vdW-DF1 (vdWDF2) are compared in the top (bottom) panel. Each point represents a binding energy for a pair of molecules in the S22 data set. The binding energies are for sfd-vdW-DF and sc-vdW-DF for vdW-DF1 and vdW-DF2, and as calculated with a quantum chemistry (QC) method. The sc and QC results are taken from Ref. 19, QC results are originally from Ref. 83. All sfd and sc calculations are carried out at the sc-vdW-DF1 or sc-vdW-DF2 binding separations (Ref. 19). The performance of the sfd scheme using vdW-DF is excellent for S22, both as compared with sc-vdW-DF results and to QC results.

Appendix derives the following explicit expressions for the linear-order error of using the sfd scheme:

$$
\int d^{3} \mathbf{r}\left\{n_{\mathrm{sc}}^{v}(\mathbf{r})-n_{\mathrm{sfd}}^{g}(\mathbf{r})\right\}\left\{\mu_{\mathrm{xc}}^{v}\left[n_{\mathrm{sfd}}^{v}\right](\mathbf{r})-\mu_{\mathrm{xc}}^{g}\left[n_{\mathrm{sfd}}^{g}\right](\mathbf{r})\right\} .
$$

This linear error, which is proportional to the difference between the exchange-correlation potentials $\mu_{\mathrm{xc}}$, makes us expect that the sfd scheme is in general less accurate than a Harris scheme. However, if exchange-correlation potentials are similar, the linear-order error will be small. For sfd-vdW-DF 
based on GGA KS calculations, error contributions will arise both from substituting the semilocal exchange correlation and from non-self-consistently introducing nonlocal correlation.

The promising results we here find for the sfd scheme using vdW-DF for molecular dimers indicate that the sfd error term is in fact small for many supramolecular systems. We note that nsc-vdW-DF also has a linear error term and that it does in general produce good agreement with sc-vdW-DF.

\section{COMPUTATIONAL DETAILS}

To generate input densities and for the nsc kinetic-energy evaluation, we use the plane-wave DFT code DACAPO. ${ }^{77}$ This code was chosen because it is straightforward to set the electronic densities equal to the sum of molecular (frozen input) densities $n_{\text {sfd }}=n_{1}+n_{2}$ through an external manipulation in ASE (Ref. 79) and thus to prepare the sfd calculations.

The densities and kinetic energy is evaluated using the PBE version $^{80}$ of GGA, relying on Vanderbilt ultrasoft pseudopotentials. We use plane-wave and density-sampling cutoffs of $500 \mathrm{eV}$. This cutoff choice has been used in many similar calculations ${ }^{28,29,42}$ and gives a relatively dense sampling of the density grid used to evaluate the nonlocal correlation. As long as the reference calculations have the same grid-sampling density, here secured by using the same size of the unit cell, the nonlocal correlation energy is typically converged to within about $1 \mathrm{meV}$.

The nonlocal correlation energy is evaluated in a postprocessing procedure both for DFT and sfd calculations. We rely on an efficient in-house real-space code, further described in Ref. 29. This code is available upon request. A radius cutoff of $6 \AA$ is used for dense (full) sampling of the grid and a cutoff of $26 \AA$ is used for sparse (double-spaced) sampling of the grid. The semilocal exchange-correlation contributions to the vdW-DF total energy function are evaluated in DACAPO.

\section{ASSESSING THE SFD SCHEME FOR SELECTED DIMERS}

Four molecular pairs, depicted in the upper panel of Fig. 3, have been chosen for a detailed comparison between the sfd scheme and regular DFT calculations. The next section describes results for the larger S22 data set of molecular dimers used for an overall organic-molecule assessment of the method.

The first of our test systems is a hydrogen fluoride (HF) dimer in parallel configuration. This configuration is not the energetically optimal one,${ }^{81,82}$ but is chosen as a representative for systems with large dipole-dipole interactions. The second is a molecular dimer of $\mathrm{HF}$ and benzene where the hydrogen of $\mathrm{HF}$ points toward the center of a benzene molecule. Thus, one molecule has zero and the other a large dipole moment. The third is a benzene dimer in parallel sandwich configuration. The binding in this system is dominated by vdW forces. This system is representative of a dilute sparse-matter system, like a gas. The fourth system, a dimer of C60 with hexagonal rings facing each other, is also one where the binding is dominated by $\mathrm{vdW}$ forces. But because of the large size of C60, this attraction is much stronger than the one of the benzene dimer. ${ }^{74}$ This
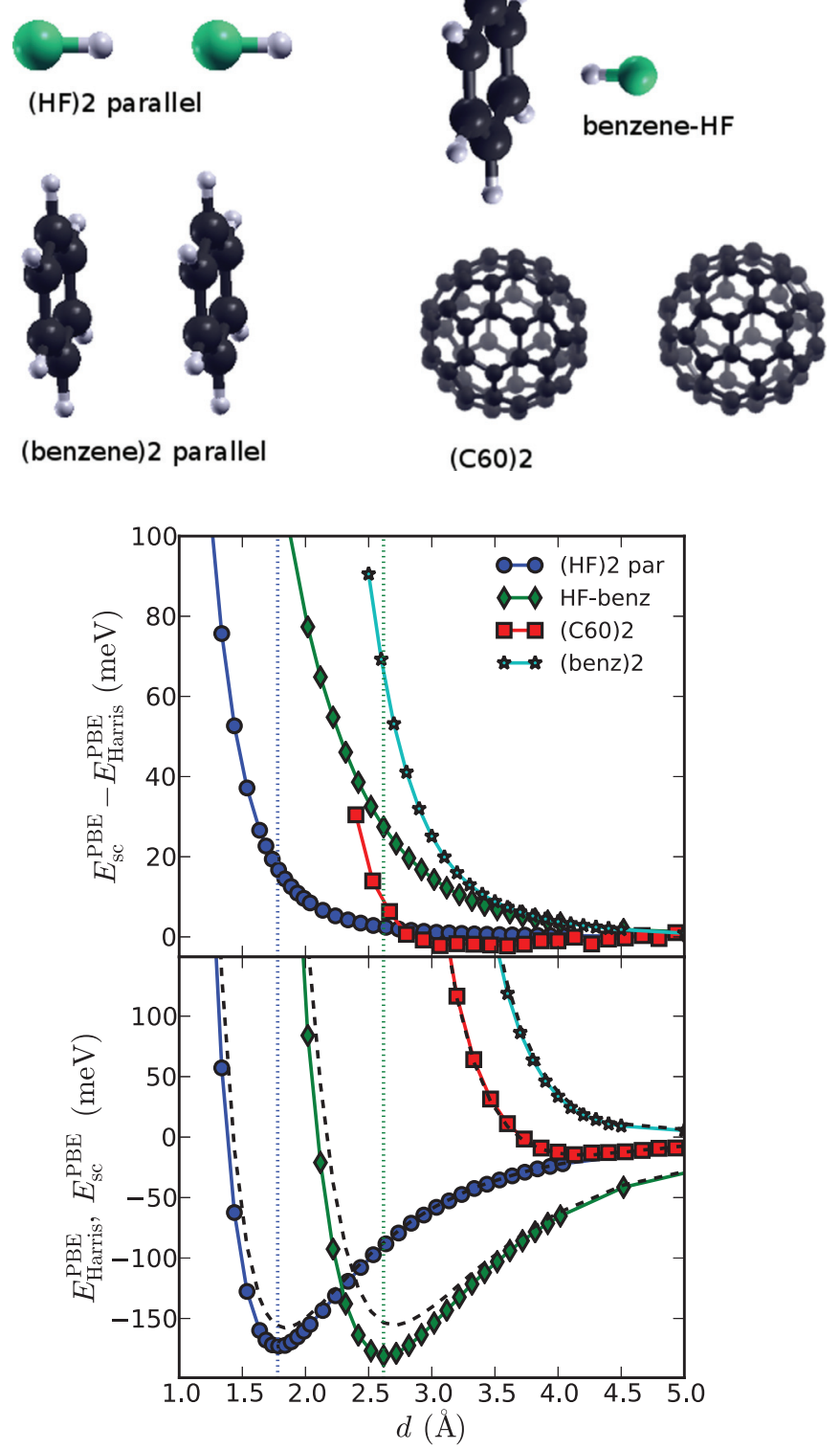

FIG. 3. (Color online) A comparison between the Harris scheme and sc DFT, for GGA calculations with the PBE functional, for four different molecular pair configurations identified in the top panel and the text. The middle panel shows the difference between the interaction energy of the sc and Harris results. The lower panel shows the interaction curves using the Harris scheme (full curve) and the sc result (dashed curve). The abscissa label $d$ denotes the separation between the closest atoms in separate molecules. The two curves involving the highly polar HF exhibit a significant binding. The dotted vertical lines indicate the GGA minimum. The Harris estimate gives the largest overestimation (16\%) for the HF-benzene interaction curve.

system is therefore more representative of compact molecular complexes that arise in bulk sparse matter.

\section{A. Regular Harris scheme for GGA-PBE calculations}

We first describe and illustrate the regular Harris scheme at the GGA level. DFT calculations with GGA are generally 


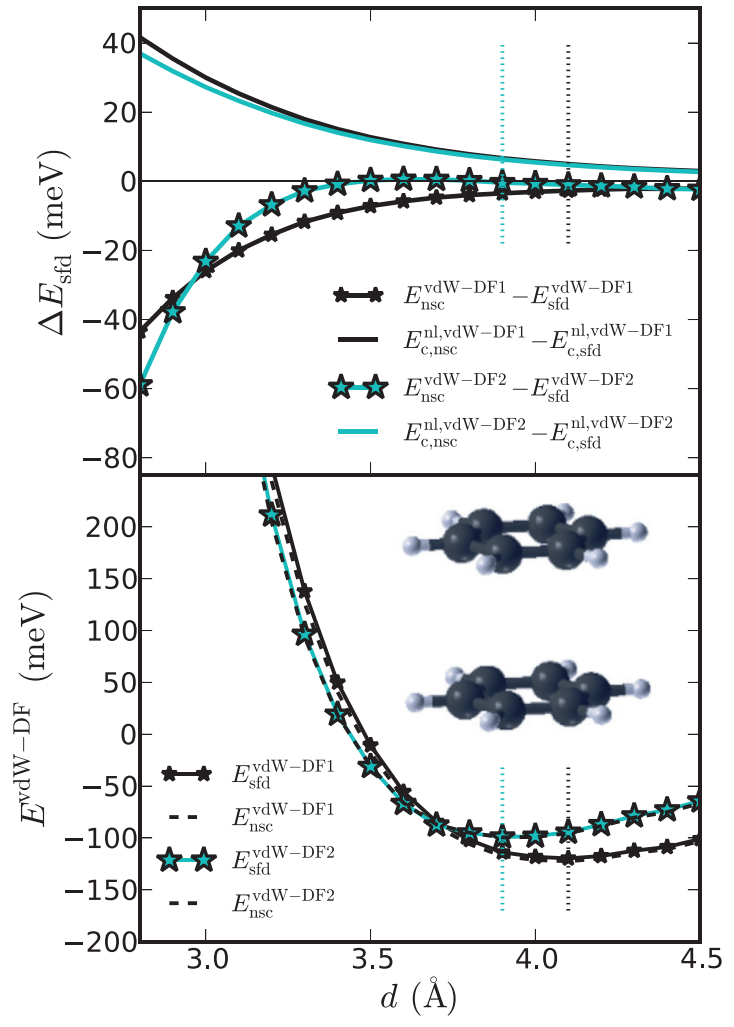

FIG. 4. (Color online) Comparison between the sfd-vdW-DF scheme and nsc-vdW-DF for a benzene dimer. The top panel shows the energetic difference between these two procedures for the total interaction energy of vdW-DF1 (black curve with small stars) and vdW-DF2 (cyan/light curves with large stars), and for the nonlocal correlation components (upper curves). The bottom panel compares the sfd-vdW-DF1 (black with small stars) and sfdvdW-DF2 (cyan/light with large stars) curves against nsc-vdW-DF (dashed curve). The somewhat larger shift, for most separations, in the nonlocal correlation energy when using the sfd scheme in place of nsc-vdW-DF energy indicates a partial cancellation of the density sensitivity of the nonlocal and semilocal components.

inadequate for describing structural properties of sparse, weakly interacting, molecular systems. Nevertheless, we find it instructive to illustrate that the GGA-level Harris scheme generally reproduces sc GGA calculations, including its sparse-matter limitations.

Figure 3 compares the interaction curves for the four different molecular pairs as obtained with DFT and the Harris scheme. The PBE version of the GGA exchange-correlation functional is used. Only the HF dimer and the HF-benzene pair show an appreciable binding of, respectively, 173 and $180 \mathrm{meV}$ using the Harris scheme for PBE and 158 and $156 \mathrm{meV}$ using sc PBE calculations. For the parallel HF dimer system, which is dominated by dipole-dipole interactions, the Harris calculation overestimates the binding energy by $9 \%$ compared to regular GGA DFT calculations. For the HF-benzene system, the scheme overestimates the binding energy by $17 \%$.

The discrepancies between the two methods can be understood from the significant dipole moment induced by the binding. At optimal separation (in the selected configurations), a dipole of $0.12 \mathrm{e} \AA$ is induced for the HF dimer, while one of $0.15 \mathrm{e} \AA$ is induced for the HF-benzene pair. These induced dipole moments are comparable to the dipole moment of the HF molecule itself $(0.39 \mathrm{e} \AA)$. It is clear that molecular pairs involving HF molecules serve as tough tests for the feasibility of the Harris functional scheme.

For the two systems dominated by the vdW forces, the Harris-based curves closely follow the DFT-based ones, which exhibit shallow minimums.

\section{B. Systems dominated by vdW attraction}

Figure 4 shows the comparison between the sfd and nscvdW-DF calculations for the benzene dimer. The difference between the dashed and the full curves in the lower panel is barely distinguishable. At binding separation, the sfd result is $2 \%$ below the nsc result for vdW-DF1 and merely $0.4 \%$ for vdW-DF2. The upper panel of Fig. 4 shows that the nonlocal energy is somewhat affected by using the sfd density $n_{\text {sfd }}=n_{1}+n_{2}$ in place of the one determined with a full GGA calculation $n_{\mathrm{nsc}}$. There is some error cancellation between the energetic shifts arising from the nonlocal correlation energy and the combined shifts arising from the other terms: the shifts obtained with the sfd scheme overestimate the nonlocal interaction energy, while the magnitude of the binding energy is underestimated. For vdW-DF2 this error cancellation is close to exact in a wide region around the binding separation. For shorter separation between the molecules, corresponding to a larger density overlap, the results of the nsc and sfd schemes differ more.

Figure 5 compares the two methods for a C60 dimer in the same fashion as for the benzene dimer. In this case, the

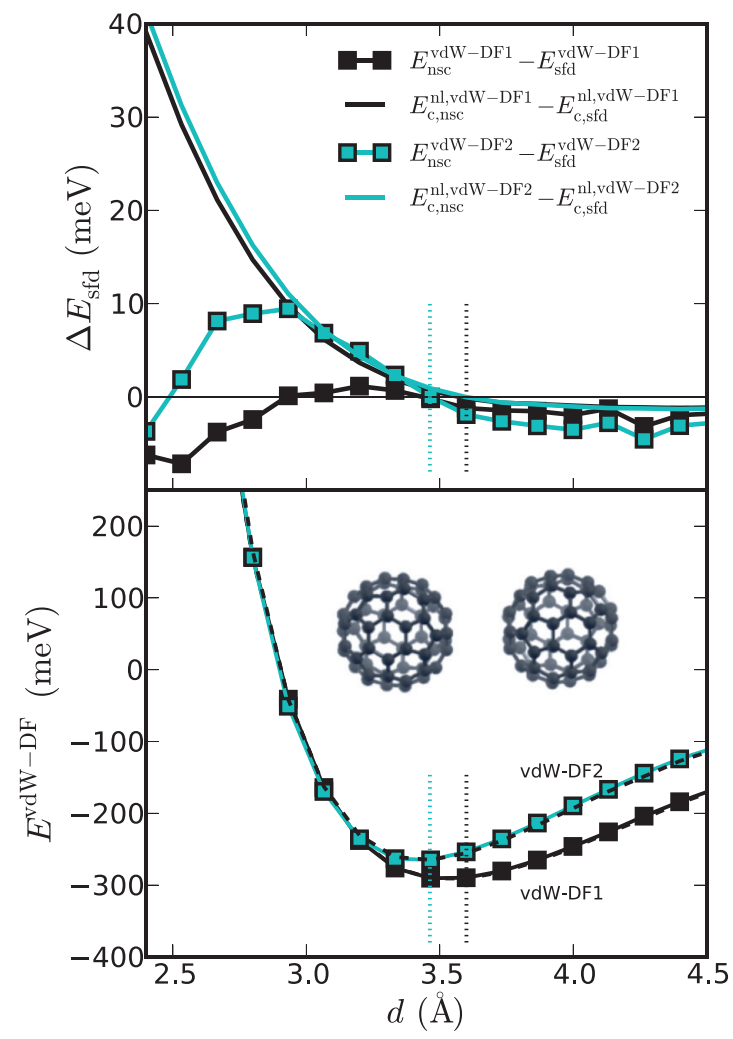

FIG. 5. (Color online) The sfd-vdW-DF description of binding in the C60 dimer; legends and details as described in Fig. 4. 


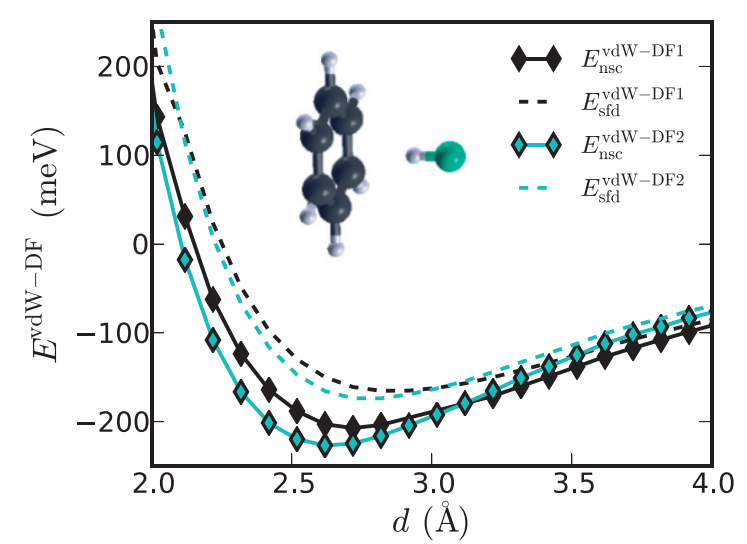

FIG. 6. (Color online) The sfd-vdW-DF description of binding between a HF molecule and benzene. Legends and details as in the lower panel of Fig. 4. The sizable discrepancy between the sfd and sc calculations in the PBE calculations, shown in Fig. 3, carries through to the case of ( $\mathrm{sfd}$ and nsc) vdW-DF calculations. The nonlocal correlation has only a tiny effect on the discrepancy.

sfd-vdW-DF1 underestimates the binding energy by as little as $0.2 \%$, while sfd-vdW-DF2 is spot on (within about $0.05 \mathrm{meV}$ ). This striking coincidence (arising from error cancellation) is likely fortuitous: the sfd-vdW-DF calculations can, depending on the separation, both overestimate and underestimate the nsc-vdW-DF interaction energy.

The benzene and C60 dimer calculations indicate that the sfd scheme is an appropriate method to accelerate the evaluation of interaction energies in systems dominated by $\mathrm{vdW}$ interactions.

\section{System with large induced charge: HF interacting with benzene}

In the HF-benzene system the vdW forces contribute to the binding alongside electrostatic effects. The nsc-vdW-DF2 predicts a binding energy of $174 \mathrm{meV}$ compared to that of $155 \mathrm{meV}$ with the sc PBE calculations (in Fig. 3).

Figure 6 shows the interaction curves obtained with sfd and nsc vdW-DF calculations for the HF-benzene system. For this system, we find that the vdW-DF2 produces a larger binding energy than vdW-DF1, which is opposite to that of the benzene and C60 dimers. This switching is related to the less repulsive exchange ${ }^{25}$ and a less attractive nonlocal correlation account ${ }^{47}$ of vdW-DF2 compared to vdW-DF1. Since the considered system is smaller in size than the benzene dimer, the magnitude of the nonlocal correlation is smaller and thus the balance between the repulsive and attractive terms is shifted.

The difference between the sfd and nsc vdW-DF calculations increases to as much as $30 \%$ for vdW-DF2, compared to $17 \%$ for the sfd and sc PBE calculations. The discrepancy is somewhat smaller for vdW-DF1. The inaccuracy of the sfd scheme is not due to the shift in the nonlocal correlation, which contributes only $4 \mathrm{meV}$ in the opposite direction of the total shift of $-60 \mathrm{meV}$.

\section{S22-BASED ASSESSMENT OF THE SFD-VDW-DF PERFORMANCE}

Figures 1 and 2 and Table I present a summary of the assessment of the accuracy of the sfd-vdW-DF scheme for the $\mathrm{S} 22$ benchmark set. ${ }^{76}$ Here, we provide some details not discussed in Sec. II B.

TABLE I. Interaction energies for pairs of small molecules from the S22 data set. Quantum chemistry (QC) results from Ref. 83, the self-consistent (sc) vdW-DF1 and vdW-DF2 results are from Ref. 19. All energies in meV/dimer.

\begin{tabular}{|c|c|c|c|c|c|c|c|c|}
\hline No. & Duplex & $E_{\mathrm{sfd}}^{\mathrm{vdW}-\mathrm{DF} 1}$ & $E_{\mathrm{nsc}}^{\mathrm{vdW}-\mathrm{DF} 1}$ & $E_{\mathrm{sc}}^{\mathrm{vdW}-\mathrm{DF} 1}$ & $E_{\mathrm{sfd}}^{\mathrm{vdW}-\mathrm{DF} 2}$ & $E_{\mathrm{nsc}}^{\mathrm{vdW}-\mathrm{DF} 2}$ & $E_{\mathrm{sc}}^{\mathrm{vdW}-\mathrm{DF} 2}$ & QC \\
\hline 1 & Ammonia dimer & 118 & 111 & 115 & 136 & 126 & 134 & 137 \\
\hline 2 & Water dimer & 186 & 171 & 185 & 220 & 201 & 218 & 218 \\
\hline 3 & Formic acid dimer & 736 & 680 & 690 & 817 & 745 & 766 & 815 \\
\hline 4 & Formamide dimer & 610 & 560 & 587 & 684 & 619 & 655 & 699 \\
\hline 5 & Uracil dimer & 807 & 749 & 767 & 876 & 805 & 832 & 897 \\
\hline 6 & 2-pyridoxine-2-aminopyridine & 671 & 623 & 639 & 728 & 661 & 687 & 737 \\
\hline 7 & Adenine-thymine & 652 & 619 & 609 & 716 & 661 & 660 & 726 \\
\hline 8 & Methane dimer & 38 & 39 & 36 & 29 & 30 & 30 & 23 \\
\hline 9 & Ethene dimer & 70 & 67 & 64 & 65 & 61 & 65 & 65 \\
\hline 10 & Benzene-methane & 72 & 70 & 68 & 64 & 62 & 63 & 63 \\
\hline 11 & Benzene dimer (slip parallel) & 136 & 141 & 136 & 120 & 124 & 123 & 114 \\
\hline 12 & Pyrazine dimer & 189 & 188 & 185 & 178 & 177 & 177 & 182 \\
\hline 13 & Uracil dimer (stacked) & 414 & 396 & 403 & 412 & 391 & 402 & 422 \\
\hline 14 & Indole-benzene (stacked) & 231 & 232 & 206 & 234 & 199 & 197 & 199 \\
\hline 15 & Adenine-thymine (stacked) & 466 & 456 & 461 & 467 & 457 & 466 & 506 \\
\hline 16 & Ethene-ethine & 74 & 68 & 69 & 74 & 67 & 70 & 65 \\
\hline 17 & Benzene-water & 142 & 125 & 124 & 148 & 126 & 129 & 143 \\
\hline 18 & Benzene-ammonia & 104 & 97 & 94 & 101 & 91 & 92 & 101 \\
\hline 19 & Benzene-HCN & 194 & 162 & 166 & 198 & 159 & 170 & 197 \\
\hline 20 & Benzene dimer ( $\mathrm{T}$ shape) & 120 & 115 & 113 & 108 & 101 & 105 & 118 \\
\hline 21 & Indole-benzene (T shape) & 240 & 210 & 214 & 230 & 192 & 206 & 243 \\
\hline 22 & Phenol dimer & 267 & 252 & 254 & 290 & 270 & 279 & 307 \\
\hline
\end{tabular}


Table I presents the specific numbers of our comparison of sc, nsc, and sfd results using vdW-DF1 and vdW-DF2 for interaction energies for the $\mathrm{S} 22$ set of molecular dimers. These interaction energies are evaluated at the binding separation (identified in Ref. 19) that minimizes respectively the sc-vdWDF1 and sc-vdW-DF2 interaction energy. The quantum chemistry results are from Ref. 83. This S22-based benchmarking can be improved by comparing the full binding-energy curves for each of the computational approaches. Here, we merely complement our earlier assessment based on binding curves of the four selected molecular dimers (upper panel of Fig. 3) with statistics for the S22 dimers.

Systems 1-7 of Table I may be labeled hydrogen-bonding dominated, while 8-15 may be labeled dispersion dominated, and the remainder mixed. The table reveals that sfd results compare well with nsc results for dispersion-dominated systems, while the biggest discrepancies arise for systems dominated by hydrogen bonds. This result agrees well with our analysis based on Figs. 3-6.

Figure 2 conveys an overview and sense of the quality of the sfd calculations compared to nsc and sc ones. The figure and table show that nsc and sc calculations are very similar. The inaccuracy introduced is overall smaller than the difference between sc-vdW-DF1 and quantum chemistry (QC) results, while the inaccuracy is about equal to the difference between QC and vdW-DF2 results. Note that vdW-DF2 has better performance for the S22 data set than vdW-DF1. The inaccuracy introduced by using sfd does not necessarily worsen results compared to the QC reference results, often fortuitous error cancellations occur.

\section{DISCUSSION OF SPEEDUP}

The speedup gained when using the sfd calculations with DACAPO is clearly evident yet somewhat modest. Computational costs are reduced by $40 \%$ and $55 \%$ for the benzene and C60 dimer, respectively, when using standard cutoffs with a minimal number of bands. Considering that this software usually requires about 20 electronic iterations to converge at these system sizes (but more for large systems), this gain is less than one might anticipate. ${ }^{68}$ However, it should be kept in mind that standard software such as DACAPO (that we here use) has been subjected to intense efforts to optimize its ability to simultaneously solve the problem of charge relaxation and determination of the KS eigenvalues. What formally constitutes Harris calculations in that code is today primarily used to obtain accurate values for the $\mathrm{KS}$ eigenvalues. ${ }^{78}$ We do not desire such enhanced accuracy for an actual sfd-vdW-DF study. The fact that the sfd-vdW-DF is still faster than nsc-vdW-DF is therefore promising. Furthermore, since the performance, documented here, is excellent for many molecular systems, there is room for more compromise on accuracy.

The sfd scheme has the greatest potential for accelerating calculations of systems consisting of many fragments since sc charge relations are only performed for the separate fragments which are much smaller than the full system. For sparse-matter systems, a fragment will either consist of a molecule or a small cluster. Here, we have only tested the feasibility of the sfd scheme for two molecules, but the method is straightforward to use for many fragments.

\section{SUMMARY AND OUTLOOK}

A Harris-type approximation, that is, to only calculate the density self-consistently for separate building blocks and estimate the total energy without relaxing the density, can work reasonably well for describing the kinetic-energy effects of forming covalent bonds of atoms in some molecules. ${ }^{68,70-72}$ We can thus expect it to have a good chance of describing the simpler kinetic-energy repulsion (steric hindrance) of molecules in supramolecular systems. Here, we have put this expectation to the test.

Our results indicate that the sfd scheme, a generalization of the Harris scheme, is promising for describing supramolecular systems bonded primarily by vdW forces. However, if one or more fragments are highly polar, this comes at the cost of accuracy.

Having established the promise of the sfd scheme for systems bound by vdW forces, the next step would be to investigate if the scheme can be further accelerated, in particular for large supramolecular systems, potentially consisting of many molecules.

\section{ACKNOWLEDGMENTS}

The authors thank K. Lee, P. Canepa, and T. Thonhauser for discussions. Partial support from the Swedish Research Council (VR) (two grants) and the Chalmers Area of Advance "Materials" is acknowledged. The computations were performed on high-performance computing resources provided by the Swedish National Infrastructure for Computing (SNIC) at the C3SE and HPC2N metanodes.

\section{APPENDIX : HARRIS-TYPE EVALUATION SCHEMES}

This appendix presents formal equations for the Harris functional and the more generalized sfd scheme. Linear-order error estimates are derived. The starting point is the $\mathrm{KS}$ equations, which will be used as a reference. We show that the regular Harris scheme has a second-order error in the density shift and that the sfd scheme has a first-order error term that is proportional to the shift in the exchange-correlation potential. Non-self-consistent vdW-DF calculations can be seen as sfd calculations in the limit of one fragment.

\section{Self-consistent KS calculations}

The KS total energy functional can be written as ${ }^{68}$

$$
\begin{aligned}
E_{\mathrm{KS}}[n]= & T_{0}+\int d^{3} \mathbf{r} n(\mathbf{r})\left[\frac{1}{2} \phi[n](\mathbf{r})+V_{\mathrm{ext}}(\mathbf{r})\right] \\
& +E_{\mathrm{xc}}[n]+E_{N} .
\end{aligned}
$$

Here, $T_{0}$ is the kinetic energy of the single-particle Kohn-Sham wave functions, and

$$
\phi[n](\mathbf{r})=\int d^{3} \mathbf{r}^{\prime} \frac{n\left(\mathbf{r}^{\prime}\right)}{\left|\mathbf{r}-\mathbf{r}^{\prime}\right|}
$$

is the electrostatic potential of the electrons. $V_{\text {ext }}(\mathbf{r})$ is the external potential that is typically specified by the ionic 
positions. $E_{\mathrm{xc}}$ is the exchange-correlation functional and $E_{N}$ is the internuclear repulsion term.

The KS scheme proceeds by determining the single-particle solutions of an effective eigenvalue problem

$$
\left\{-\frac{1}{2} \nabla^{2}+V_{\text {eff }}[n](\mathbf{r})-\epsilon_{\lambda}\right\} \psi_{\lambda}(\mathbf{r})=0
$$

defined by the density-dependent effective potential

$$
V_{\mathrm{eff}}[n](\mathbf{r}) \equiv V_{\mathrm{ext}}(\mathbf{r})+\phi[n](\mathbf{r})+\mu_{\mathrm{xc}}[n](\mathbf{r}) .
$$

The exchange-correlation potential $\mu_{\mathrm{xc}}$ is given as a functional derivative of the exchange-correlation functional

$$
\mu_{\mathrm{xc}}[n](\mathbf{r}) \equiv \frac{\delta E_{\mathrm{xc}}[n]}{\delta n(\mathbf{r})} .
$$

Self-consistency is enforced by demanding that the singleparticle description of the electron density

$$
\tilde{n}(\mathbf{r})=\sum_{\lambda}^{\mathrm{occ}}\left|\psi_{\lambda}(\mathbf{r})\right|^{2}
$$

matches the density that specifies the effective single-particle potential (A3).

This procedure provides the self-consistent density $n_{\mathrm{sc}}$ and the single-particle energies $\epsilon_{\lambda}$. The total energy is obtained by summing over the set of occupied, single-particle energies $\epsilon_{\lambda}$ and correcting for a double counting of the electron-electron interaction energy,

$$
\begin{aligned}
E_{\mathrm{KS}}(d)= & \sum_{\lambda}^{\mathrm{occ}} \epsilon_{\lambda}-\int d^{3} \mathbf{r} n_{\mathrm{sc}}(\mathbf{r})\left\{\frac{1}{2} \phi\left[n_{\mathrm{sc}}\right](\mathbf{r})+\mu_{\mathrm{xc}}\left[n_{\mathrm{sc}}\right](\mathbf{r})\right\} \\
& +E_{\mathrm{xc}}\left[n_{\mathrm{sc}}\right]+E_{N}(d) .
\end{aligned}
$$

Here, an atomic-geometry-specific parameter $d$ has been introduced. The KS energy acquires a minimum at the correct ground-state density $n_{\mathrm{sc}}$ :

$$
E_{\mathrm{KS}}\left[n_{\mathrm{sc}}+\delta n\right]=E_{\mathrm{KS}}\left[n_{\mathrm{sc}}\right]+C(\delta n)^{2}, \quad C>0 .
$$

\section{Regular Harris scheme}

The Harris scheme avoids updating the electron density when estimating the interaction energy. The input is given as a superposition of self-consistently determined densities for each fragment: $n_{\mathrm{sfd}} \equiv \sum_{i} n_{\mathrm{sc}, i}$. The total energy estimate can be expressed as ${ }^{68}$

$$
\begin{aligned}
& E_{\text {Harris }}^{g}(d) \\
& \equiv \sum_{\lambda}^{\text {occ }} \bar{\epsilon}_{\lambda}^{g}-\int d^{3} \mathbf{r} n_{\mathrm{sfd}}^{g}(\mathbf{r})\left\{\frac{1}{2} \phi\left[n_{\mathrm{sfd}}^{g}\right](\mathbf{r})+\mu_{\mathrm{xc}}^{g}\left[n_{\mathrm{sfd}}^{g}\right](\mathbf{r})\right\} \\
& \quad+E_{\mathrm{xc}}^{g}\left[n_{\mathrm{sfd}}^{g}\right]+E_{N}(d) .
\end{aligned}
$$

The parameter $g$ is a label for the exchange-correlation functional used (suppressed in the previous section). In the Harris estimate (A9), the eigenvalues $\bar{\epsilon}_{\lambda}^{g}$ are the single-particle energies calculated within the Harris "one-shot" (no density update) calculation of the frozen input superposition density $n_{\text {sfd }}^{g}$ using the effective potential

$$
V_{\mathrm{eff}}^{g}\left[n_{\mathrm{sfd}}^{g}\right](\mathbf{r})=V_{\mathrm{ext}}(\mathbf{r})+\phi\left[n_{\mathrm{sfd}}^{g}\right](\mathbf{r})+\mu_{\mathrm{xc}}^{g}\left[n_{\mathrm{sfd}}^{g}\right](\mathbf{r}) .
$$

The Harris scheme rests on the assumption that the density shift $\delta n=n_{\mathrm{sc}}-n_{\mathrm{sfd}}$ produces only a small change in the effective potential

$$
\begin{aligned}
\Delta V_{\mathrm{eff}}(\mathbf{r})= & \phi\left[n_{\mathrm{sc}}^{g}\right](\mathbf{r})-\phi\left[n_{\mathrm{sfd}}^{g}\right](\mathbf{r}) \\
& +\mu_{\mathrm{xc}}^{g}\left[n_{\mathrm{sc}}^{g}\right](\mathbf{r})-\mu_{\mathrm{xc}}^{g}\left[n_{\mathrm{sfd}}^{g}\right](\mathbf{r}),
\end{aligned}
$$

so that we can expand the difference in KS and Harris estimates for the single-particle energy sum

$$
\sum_{\lambda}^{\mathrm{occ}} \epsilon_{\lambda}-\sum_{\lambda^{\prime}}^{\mathrm{occ}} \bar{\epsilon}_{\lambda^{\prime}}=\int d^{3} \mathbf{r} n_{\mathrm{sc}}(\mathbf{r}) \Delta V_{\mathrm{eff}}(\mathbf{r})+\mathcal{O}\left(\delta n^{2}\right) .
$$

The linear term cancels out corresponding linear terms in the expansion of $E_{\mathrm{xc}}[n]$ and in the calculation of the electrostatic potential. ${ }^{68}$ This cancellation follows from

$$
\begin{aligned}
& \int d^{3} \mathbf{r} \phi\left[n_{\mathrm{sc}}\right](\mathbf{r}) n_{\mathrm{sfd}}(\mathbf{r})=\int d^{3} \mathbf{r} \phi\left[n_{\mathrm{sfd}}\right](r) n_{\mathrm{sc}}(\mathbf{r}), \\
& E_{\mathrm{xc}}\left[n_{\mathrm{sc}}^{g}\right]-E_{\mathrm{xc}}\left[n_{\mathrm{sfd}}^{g}\right] \approx \int d^{3} \mathbf{r} \delta n(\mathbf{r}) \mu_{\mathrm{xc}}^{g}\left[n_{\mathrm{sfd}}^{g}\right](\mathbf{r}) .
\end{aligned}
$$

The single-shot (no density update) Harris estimate of the total energy is variational

$$
E_{\text {Harris }}\left[n_{\text {sfd }}\right]=E_{\text {Harris }}\left[n_{\mathrm{sc}}\right]+\mathcal{O}\left(\delta n^{2}\right),
$$

but unlike the similar KS result (A8), it is not, in general, an extremum. ${ }^{68,70,84,85}$ This follows because there is no consistency between the Harris-scheme input density $n_{\text {sfd }}$ and the single-particle electron density (A6) that results with the Harris-scheme effective potential $V_{\text {eff }}\left[n_{\text {sfd }}\right](\mathbf{r})$.

\section{Formal error estimate in the sfd scheme}

In the sfd scheme, the functional $v$ used to calculate the exchange-correlation energy $E_{\mathrm{xc}}^{v}$ in the Harris estimate (A9) may differ from the functional $g$ used to obtain the input density $n_{\text {sfd }}^{g}$ and single-particle spectrum. To establish a formal relation between sc and sfd calculations, we consider the differences in sc results and sfd result. We introduce

$$
\begin{gathered}
\delta n_{\mathrm{sfd}}^{v, g} \equiv n_{\mathrm{sc}}^{v}-n_{\mathrm{sfd}}^{g}, \\
\Delta V_{\mathrm{sfd}} \equiv \phi\left[n_{\mathrm{sc}}^{v}\right]-\phi\left[n_{\mathrm{sfd}}^{g}\right]+\mu_{\mathrm{xc}}^{v}\left[n_{\mathrm{sc}}^{v}\right]-\mu_{\mathrm{xc}}^{g}\left[n_{\mathrm{sfd}}^{g}\right]
\end{gathered}
$$

to identify the changes resulting in the density and in the effective potential, respectively. As in the original Harris analysis, ${ }^{68}$ we can consider both $\delta n_{\text {sfd }}$ and $\Delta V_{\text {sfd }}$ to be small parameters and consider only linear-order terms.

The energetic shift arising from the one-particle energies is given by

$$
\sum_{\lambda}^{\mathrm{occ}} \epsilon_{\lambda}^{v}-\sum_{\lambda}^{\mathrm{occ}} \bar{\epsilon}_{\lambda}^{g} \approx \int d^{3} \mathbf{r} n_{\mathrm{sc}}^{v}(\mathbf{r}) \Delta V_{\mathrm{sfd}}(\mathbf{r}) .
$$

The shift in the exchange-correlation term is

$$
E_{\mathrm{xc}}^{v}\left[n_{\mathrm{sc}}^{v}\right]-E_{\mathrm{xc}}^{v}\left[n_{\mathrm{sfd}}^{g}\right] \approx \int d^{3} \mathbf{r} \delta n_{\mathrm{sfd}}^{v, g}(\mathbf{r}) \mu_{\mathrm{xc}}^{v}\left[n_{\mathrm{sfd}}^{v}\right](\mathbf{r}) .
$$


The formal error estimate in the sfd scheme is thus

$$
\Delta E_{\mathrm{sfd}}^{v, g}=\int d^{3} \mathbf{r} \delta n_{\mathrm{sfd}}^{v, g}(\mathbf{r})\left\{\mu_{\mathrm{xc}}^{v}\left[n_{\mathrm{sfd}}^{v}\right](\mathbf{r})-\mu_{\mathrm{xc}}^{g}\left[n_{\mathrm{sfd}}^{g}\right](\mathbf{r})\right\} .
$$

The error is linear in density, but also proportional to the shift in the exchange-correlation potential.
In the limit of $v=g$, we get the Harris functional and the two the terms in (A20) cancel. If we replace $\delta n_{\mathrm{sfd}}^{v, g} \rightarrow n_{\mathrm{sc}}^{v}-$ $n_{\mathrm{sc}}^{g}$, we obtain the formal error estimate of standard non-selfconsistent vdW-DF. This procedure can be seen as a special one-fragment limit of sfd. Although this procedure also has a linear error term in the density shift, the density shift will typically be small.
*Corresponding author; schroder@chalmers.se

${ }^{1}$ K. Burke, J. Chem. Phys. 136, 150901 (2012).

${ }^{2}$ D. C. Langreth, B. I. Lundqvist, S. D. Chakarova-Käck, V. R. Cooper, M. Dion, P. Hyldgaard, A. Kelkkanen, J. Kleis, L. Kong, S. Li, P. G. Moses, E. Murray, A. Puzder, H. Rydberg, E. Schröder, and T. Thonhauser, J. Phys.: Condens. Matter 21, 084203 (2009). ${ }^{3}$ G. D. Mahan, J. Chem. Phys. 43, 1569 (1965).

${ }^{4}$ K. Rapcewicz and N. W. Ashcroft, Phys. Rev. B 44, 4032 (1991). ${ }^{5}$ Y. Andersson, D. C. Langreth, and B. I. Lundqvist, Phys. Rev. Lett. 76, 102 (1996).

${ }^{6}$ H. Rydberg, B. I. Lundqvist, D. C. Langreth, and M. Dion, Phys. Rev. B 62, 6997 (2000).

${ }^{7}$ D. C. Langreth, M. Dion, H. Rydberg, E. Schröder, P. Hyldgaard, and B. I. Lundqvist, Int. J. Quantum Chem. 101, 599 (2005).

${ }^{8}$ X. Wu, M. C. Vargas, S. Nayak, V. Lotrich, and G. Scoles, J. Chem. Phys. 115, 8748 (2001).

${ }^{9}$ M. Elstner, P. Hobza, T. Frauenheim, S. Suhai, and E. Kaxiras, J. Chem. Phys. 114, 5149 (2001).

${ }^{10}$ S. Grimme, J. Comput. Chem. 25, 1463 (2004); J. Chem. Phys. 124, 034108 (2006); S. Grimme, J. Antony, S. Ehrlich, and H. Krieg, ibid. 132, 154104 (2010).

${ }^{11}$ A. Tkatchenko and M. Scheffler, Phys. Rev. Lett. 102, 073005 (2009); V. G. Ruiz, W. Liu, E. Zojer, M. Scheffler, and A. Tkatchenko, ibid. 108, 146103 (2012); A. Tkatchenko, R. A. DiStasio, Jr., R. Car, and M. Scheffler, ibid. 108, 236402 (2012).

${ }^{12}$ H. Rydberg, M. Dion, N. Jacobson, E. Schröder, P. Hyldgaard, S. I. Simak, D. C. Langreth, and B. I. Lundqvist, Phys. Rev. Lett. 91, 126402 (2003).

${ }^{13}$ M. Dion, H. Rydberg, E. Schröder, D. C. Langreth, and B. I. Lundqvist, Phys. Rev. Lett. 92, 246401 (2004); 95, 109902(E) (2005).

${ }^{14}$ T. Thonhauser, V. R. Cooper, S. Li, A. Puzder, P. Hyldgaard, and D. C. Langreth, Phys. Rev. B 76, 125112 (2007).

${ }^{15}$ E. R. Johnson and A. D. Becke, J. Chem. Phys. 122, 154104 (2005); 123, 024101 (2005); 123, 154101 (2005).

${ }^{16}$ O. A. Vydrov and T. V. Voorhis, J. Chem. Phys. 130, 104105 (2009).

${ }^{17}$ O. A. Vydrov and T. V. Voorhis, Phys. Rev. Lett. 103, 063004 (2009).

${ }^{18}$ O. A. Vydrov and T. V. Voorhis, J. Chem. Phys. 133, 244103 (2010).

${ }^{19}$ K. Lee, E. D. Murray, L. Kong, B. I. Lundqvist, and D. C. Langreth, Phys. Rev. B 82, 081101(R) (2010).

${ }^{20}$ H. Rydberg, Ph.D. thesis, Chalmers University of Technology, 2001; P. Hyldgaard, K. Berland, H. Rydberg, E. Schröder, and B. I. Lundqvist (unpublished).

${ }^{21}$ M. Dion, Ph.D. thesis, Rutgers University, 2004.

${ }^{22}$ O. Gunnarsson and B. I. Lundqvist, Phys. Rev. B 13, 4274 (1976).

${ }^{23}$ D. C. Langreth and J. P. Perdew, Phys. Rev. B 15, 2884 (1977).

${ }^{24}$ Y. Zhang and W. Yang, Phys. Rev. Lett. 80, 890 (1998).

${ }^{25}$ E. D. Murray, K. Lee, and D. C. Langreth, J. Chem. Theory Comput. 5, 2754 (2009).
${ }^{26}$ E. Ziambaras, J. Kleis, E. Schröder, and P. Hyldgaard, Phys. Rev. B 76, 155425 (2007).

${ }^{27}$ E. Londero and E. Schröder, Phys. Rev. B 82, 054116 (2010).

${ }^{28} \mathrm{~K}$. Berland and P. Hyldgaard, J. Chem. Phys. 132, 134705 (2010).

${ }^{29}$ K. Berland, Ø. Borck, and P. Hyldgaard, Comput. Phys. Commun. 182, 1800 (2011).

${ }^{30}$ E. Londero and E. Schröder, Comput. Phys. Commun. 182, 1805 (2011).

${ }^{31}$ J. Rohrer and P. Hyldgaard, Phys. Rev. B 83, 165423 (2011).

${ }^{32}$ G. Graziano, J. Klimeš, F. Fernandez-Alonso, and A. Michaelides, J. Phys.: Condens. Matter 24, 424216 (2012).

${ }^{33}$ T. Björkman, A. Gulans, A. V. Krasheninnikov, and R. M. Nieminen, J. Phys.: Condens. Matter 24, 424218 (2012).

${ }^{34}$ N. Nijem, L. Kong, Y. Zhao, H. Wu, J. Li, D. C. Langreth, and Y. J. Chabal, J. Am. Chem. Soc. 133, 4782 (2011); N. Nijem, P. Canepa, L. Kong, H. Wu, J. Li, T. Thonhauser, and Y. J. Chabal, J. Phys.: Condens. Matter 24, 424203 (2012).

${ }^{35}$ N. Nijem, J.-F. Veyan, L. Kong, K. Li, S. Pramanik, Y. Zhao, J. Li, D. C. Langreth, and Y. J. Chabal, J. Am. Chem. Soc. 132, 1654 (2010); Q. Li and T. Thonhauser, J. Phys.: Condens. Matter 24, 424204 (2012).

${ }^{36}$ Y. Ihm, V. R. Cooper, L. Peng, and J. R. Morris, J. Phys.: Condens. Matter 24, 424205 (2012).

${ }^{37}$ S. D. Chakarova-Käck, E. Schröder, B. I. Lundqvist, and D. C. Langreth, Phys. Rev. Lett. 96, 146107 (2006).

${ }^{38}$ S. D. Chakarova-Käck, Ø. Borck, E. Schröder, and B. I. Lundqvist, Phys. Rev. B 74, 155402 (2006).

${ }^{39}$ K. Berland, T. L. Einstein, and P. Hyldgaard, Phys. Rev. B 80, 155431 (2009); K. Toyoda, Y. Nakano, I. Harmada, K. Lee, S. Yanagisawa, and Y. Morikawa, Surf. Sci. 609, 2912 (2009); K. Lee, Y. Morikawa, and D. C. Langreth, Phys. Rev. B 82, 155461 (2010).

${ }^{40}$ I. Hamada, K. Lee, and Y. Morikawa, Phys. Rev. B 81, 115452 (2010).

${ }^{41}$ J. Carrasco, B. Santra, J. Klimeš, and A. Michaelides, Phys. Rev. Lett. 106, 026101 (2011).

${ }^{42}$ K. Berland, S. D. Chakarova-Käck, V. R. Cooper, D. C. Langreth, and E. Schröder, J. Phys.: Condens. Matter 23, 135001 (2011).

${ }^{43}$ D.-L. Chen, W. A. Al-Saidi, and J. K. Johnson, Phys. Rev. B 84, 241405(R) (2011); J. Phys.: Condens. Matter 24, 424211 (2012).

${ }^{44}$ D. Le, M. Aminpour, A. Keijna, and T. S. Rahman, J. Phys.: Condens. Matter 24, 222001 (2012).

${ }^{45}$ D. Le, A. Kara, E. Schröder, P. Hyldgaard, and T. S. Rahman, J. Phys.: Condens. Matter 24, 424210 (2012).

${ }^{46}$ E. Londero, E. K. Karlson, M. Landahl, D. Ostrovskii, J. D. Rydberg, and E. Schröder, J. Phys.: Condens. Matter 24, 424212 (2012). 
${ }^{47}$ K. Lee, A. K. Kelkkanen, K. Berland, S. Andersson, D. C. Langreth, E. Schröder, B. I. Lundqvist, and P. Hyldgaard, Phys. Rev. B 84, 193408 (2011); K. Lee, K. Berland, M. Yoon, S. Andersson, E. Schröder, P. Hyldgaard, and B. I. Lundqvist, J. Phys.: Condens. Matter 24, 424213 (2012).

${ }^{48}$ P. Lazić, N. Atodiresei, V. Caciuc, R. Brako, B. Gumhalter, and S. Blügel, J. Phys.: Condens. Matter 24, 424215 (2012).

${ }^{49}$ F. Hanke, M. S. Dyer, J. Björk, and M. Persson, and S. Blügel, J. Phys.: Condens. Matter 24, 424217 (2012).

${ }^{50}$ A. Bergvall, K. Berland, P. Hyldgaard, S. Kubatkin, and T. Löfwander, Phys. Rev. B 84, 155451 (2011).

${ }^{51}$ J. Wyrick, D.-H. Kim, D. Sun, Z. Cheng, W. Lu, Y. Zhu, K. Berland, Y. S. Kim, E. Rotenberg, M. Luo, P. Hyldgaard, T. L. Einstein, and L. Bartels, Nano Lett. 11, 2944 (2011).

${ }^{52}$ G. Li, V. R. Cooper, J.-H. Cho, S. Du, H.-J. Gao, and Z. Zhang, Phys. Rev. B 84, 241406(R) (2011).

${ }^{53}$ M. Callsen, N. Atodiresei, V. Caciuc, and S. Blügel, Phys. Rev. B 86, 085439 (2012).

${ }^{54}$ J. Kleis, B. I. Lundqvist, D. C. Langreth, and E. Schröder, Phys. Rev. B 76, 100201(R) (2007).

${ }^{55}$ V. R. Cooper, T. Thonhauser, A. Puzder, E. Schröder, B. I. Lundqvist, and D. C. Langreth, J. Am. Chem. Soc. 130, 1304 (2008).

${ }^{56}$ J. Kleis, E. Schröder, and P. Hyldgaard, Phys. Rev. B 77, 205422 (2008).

${ }^{57}$ A. Puzder, M. Dion, and D. C. Langreth, J. Chem. Phys. 124, 164105 (2006).

${ }^{58}$ S. D. Chakarova-Käck, A. Vojvodic, J. Kleis, P. Hyldgaard, and E. Schröder, New J. Phys. 12, 013017 (2012).

${ }^{59}$ R. Sabatini, E. Kücükbenli, B. Kolb, and T. Thonhauser, J. Phys.: Condens. Matter 24, 424209 (2012).

${ }^{60}$ V. R. Cooper, Phys. Rev. B 81, 161104(R) (2010).

${ }^{61}$ J. Klimeš, D. R. Bowler, and A. Michaelides, J. Phys.: Condens. Matter 22, 022201 (2010).

${ }^{62} \mathrm{BEEF}$, an approach to select the exchange choice for vdW-DF calculations to a specific target application, based on Bayesian logic and training, J. Wellendorff, K. T. Lundgaard, A. Møgelhøj, V. Petzold, D. D. Landis, J. K. Nørskov, T. Bligård, and K. W. Jacobsen, Phys. Rev. B 85, 235149 (2012).

${ }^{63}$ G. Román-Pérez and J. M. Soler, Phys. Rev. Lett. 103, 096102 (2009).

${ }^{64}$ A. Gulans, M. J. Puska, and R. M. Nieminen, Phys. Rev. B 79, 201105(R) (2009).

${ }^{65}$ Open-source tool JUNOLO for real-space and fast-Fourier transform non-self-consistent vdW-DF total-energy evaluation; P. Lazić, N. Atodiresei, M. Alaei, V. Caciuc, S. Blügel, and R. Brako, Comput. Phys. Commun. 181, 371 (2010).
${ }^{66}$ D. Nabok, P. Puschnig, and C. Ambrosch-Draxl, Comput. Phys. Commun. 182, 1657 (2011).

${ }^{67}$ Open-source tool for real-space non-self-consistent vdW-DF total-energy evaluation, a python package NSCVDWDF (available upon request) from the Chalmers vdW-DF groups, http://fy.chalmers.se/ schroder/vdWDF.

${ }^{68}$ J. Harris, Phys. Rev. B 31, 1770 (1985).

${ }^{69}$ G. D. Bellchambers and F. R. Manby, J. Chem. Phys. 135, 084105 (2011).

${ }^{70}$ W. M. C. Foulkes and R. Haydock, Phys. Rev. B 39, 12520 (1989).

${ }^{71}$ V. K. Nikulin, Zh. Tekh. Fiz. 41, 41 (1971) [Sov. Phys. Tech. Phys. 16, 28 (1971)].

${ }^{72}$ R. G. Gordon and Y. S. Kim, J. Chem. Phys. 56, 3122 (1972).

${ }^{73}$ The approach is sometimes also called the Harris-Foulkes scheme.

${ }^{74}$ K. Berland and P. Hyldgaard, Phys. Rev. B 87, 205421 (2013).

${ }^{75}$ E. Londero, P. Hyldgaard, and E. Schröder, arXiv:1304.1936.

${ }^{76}$ P. Jurečka, J. Šponer, J. Černý, and P. Hobza, Phys. Chem. Chem. Phys. 8, 1985 (2006).

${ }^{77}$ Open-source, plane-wave-based DFT code DACAPO, http://wiki.fysik.dtu.dk/dacapo.

${ }^{78} \mathrm{~A}$ single electronic iteration is here taken to mean an evaluation stage during which the external potential is kept fixed. In most DFT codes, there are several wave-function evaluation steps in each such electronic iteration. When pursuing a standard sc DFT determination, the requested accuracy on the wave-function solution remains moderate. For band-structure calculations, the DFT code is formally performing a Harris-type evaluation, but a code such as DACAPO then forces many wave-function-evaluation steps to deliver a higher accuracy on the wave functions. However, that is not the spirit of the original Harris scheme, nor do we intend this here with the proposed sfd-vdW-DF scheme.

${ }^{79}$ Python-based atomic simulation environment, see http://wiki.fysik.dtu.dk/ase/.

${ }^{80}$ J. P. Perdew, K. Burke, and M. Ernzerhof, Phys. Rev. Lett. 77, 3865 (1996).

${ }^{81}$ W. Klopper, M. Quack, and M. A. Suhm, J. Chem. Phys. 108, 10096 (1998).

${ }^{82}$ R. A. Oriani and C. P. Smyth, J. Am. Chem. Soc. 70, 125 (1948).

${ }^{83}$ T. Takatani, E. G. Hohenstein, M. Malagoli, M. S. Marshall, and C. D. Sherrill, J. Chem. Phys. 132, 144104 (2010).

${ }^{84}$ E. Zaremba, J. Phys.: Condens. Matter 2, 2479 (1990).

${ }^{85}$ B. Farid, V. Heine, G. E. Engel, and I. J. Robertson, Phys. Rev. B 48, 11602 (1993). 